\title{
Spatial correlation between the predictor variables and the weighting values calculated during the mapping of the environmental factors of mass movements in the Beni Idder region (northern Rif)
}

\author{
Ait Brahim L., Jemmah A. I., Bousta M., El Hamdouni I., Abdelouafi A., El Mahsani A., \\ Lallout I. Labraimi A.Et Merouni F.Z
}

Université Mohammed V, Faculté des sciences, Unité de recherche GEORISK, LGRN, 4 Avenue Ibn Battouta RabatAgdal, BP 1014, Rabat, Maroc.

\section{laitbrahim@gmail.com}

\begin{abstract}
The Tleta of Beni Ider region located in the SW of Tetouan ( Rif Septentrional) knows many mass instabilities. The diagnostic via the inventory, the mapping and the characterization of mass movements was made by using satellite imagery, aerial photography and field data coupled with existing documents (geological, geomorphological,...). The understanding of both their spatial distribution and the mechanism generating them, is very complex because of the existence of an important number of natural factors (geological, geomorphological, hydrological) in a relative mountainous landscape with deep valleys, steep slopes and significant elevation changes. Thus, a multidisciplinary approach was adopted to elaborate the landslide susceptibility map of the region taking into account interactions and causal relationships between the various natural parameters that tend to accentuate and aggravate the setting of landslides. The multidisciplinary database allowed us to evaluate the susceptibility thanks to a bivariate probabiliste model (Weight of Evidence). The obtained landslide susceptibility map is a major contribution to the development of urban development plans in the region.
\end{abstract}

\section{Introduction}

L'objectif de cette partie est d'élaborer le zonage de la carte de susceptibilité aux mouvements de terrain à méso-échelle (1/50 000ème) au niveau de la région de Béni Idder. Le choix de cette échelle d'analyse pour cette région a été conditionné par un certain nombre de paramètres essentiels : La présence d'une densité relativement importante et diversifiée des mouvements de terrain; c'est une zone centrale qui regroupe les cinq classes de susceptibilité identifiées. A cela, s'ajoute l'accroissement progressif de l'installation de la population le long de la route nationale $\mathrm{N}^{\circ} 2$ (Tetouan-chechaouen) et des routes secondaires (P4704, P4702, P4405, P4403 etc.) qui ne cessent de s'accroitre dans le cadre du désenclavement de cette région.

L'approche probabiliste statistique utilisée pour le zonage de la susceptibilité aux mouvements de terrain à méso-échelle (1/50 000ème) est la théorie de l'évidence (TDE) qui est une méthode bayésienne bivariée testée en contextes montagneux [1] [2] [3] [4] [5]. La démarche adoptée est représentée dans la figure.1, et peut être résumée en cinq grandes étapes : i. La sélection des différents paramètres à intégrer dans le modèle; ii. La mise en œuvre de tests pour s'assurer de la validité des paramètres sélectionnés ; iii. La réalisation d'un nombre de simulations par la théorie de l'évidence; iiii. La comparaison des résultats qui permettra d'en sélectionner la meilleure. (Fig.1)

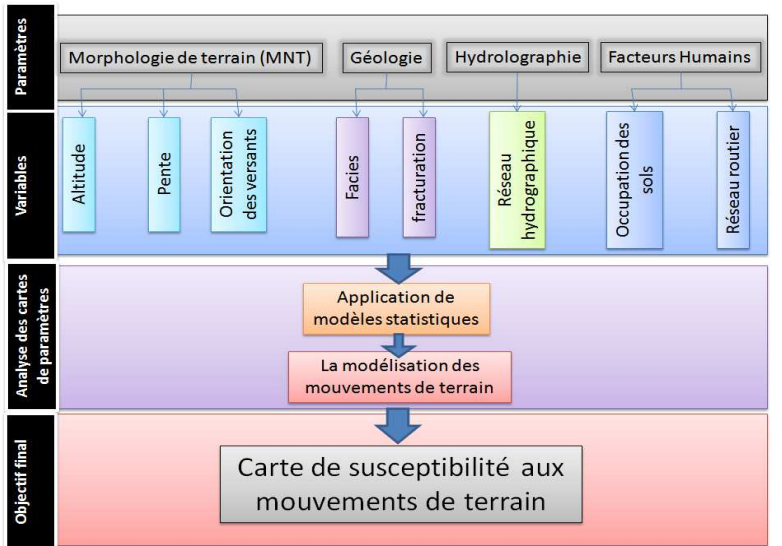

Fig.1 la démarche adoptée pour le zonage de la susceptibilité aux mouvements de terrain à méso-échelle (1/50 000ème) par la théorie de l'évidence (TDE) de la région de Béni Idder 


\section{Secteur d'étude}

\subsection{Cadre géographique}

La région d'étude est celle de Beni Idder (Rif septentrional), qui se trouvent à $35^{\circ} 24^{\prime} 0^{\prime \prime} \mathrm{N}$ et 5०30'36" E. Administrativement, elle est délimitée au Nord par Tétouan au Sud par Chefchaouene, à l'Est par Talembote et à l'Ouest par Khmis El Arous. Avec une superficie d'environ $635 \mathrm{~km}^{2}$. (Fig.2)

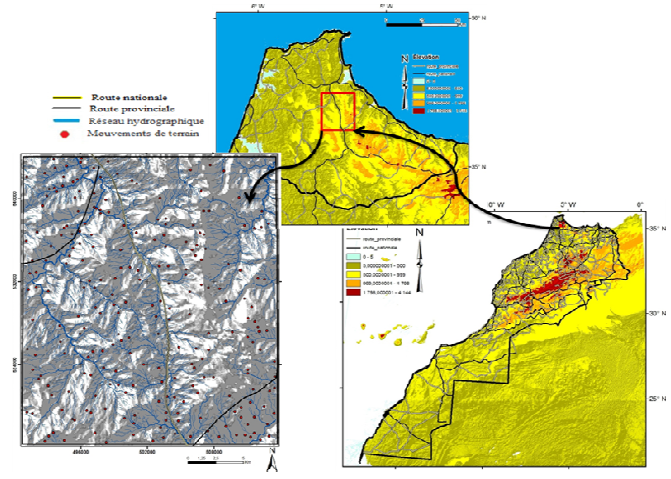

Fig.2 Situation géographique du secteur d'étude

\subsection{Cadre géomorphologique et géologique}

L'hypsométrie de la région de Tleta de Beni Ider lui imprime un caractère moyennement montagneux (1900m). Secteur caractérisé par des reliefs très accidentés et des pentes très abruptes notamment au niveau des grands contacts chevauchants entre les nappes de charriage, caractérisées par des vallées fortement encaissées, et des dénivellations relativement fortes. Une telle morphologie est le résultat de la conjugaison de plusieurs facteurs, à savoir: la lithologie complexe, le système climatique, la dynamique érosive, le style de déformations et l'activité tectoniques récente. Les unités géomorphologiques distinctes dans le secteur, ont été reconnues par [6].

De point de vue géologique; la zone d'étude appartienne au Rif septentrional. Elle est situé entre la dorsale calcaire constitué par un empilement d'écaille calcairo-dolomitique à l'est, l'unité d'Amettrasse-Bettara qui englobe des marnes d'âge éocène-oligocène au sud, et vers l'ouest apparaissent des unités de Tisirène (nappes des flysch) constitués des argilites et des marnes gréseux d'âge crétacé inférieur. La zone et couverte d'un manteau de dépôts récents constitué de coulées boueuses ,d'éboulis et d'alluvions [7].

\section{Présentation de la variable à modélisé}

Nous avons recensé et cartographié les différents mouvements de terrain de Beni Idder, en consultant les documents disponibles et en utilisant les données satellitaires (Landsat7 ETM+ haute résolution, Spot) et une série de photo-aériennes à l'échelle de 1/ 50 000eme, les résultats ont été vérifié sur le terrain (Fig.3). Nous avons classé les mouvements de terrains par typologie dans le Rif (malgré la diversité lithologique, et la complexité morphologique) en quatre classes [8]: i. Les glissements qui occupent $43,07 \%$ de la totalité des mouvements de terrain. Ils se localisent surtout dans des formations gréso-pélitiques ou des intercalations marno-calcaires. ii. Les éboulements (écroulements+ coulée de débris) qui présentent $31,46 \%$ du nombre total des mouvements recensés. Ils regroupent un ensemble de phénomènes rapides et brutaux qui affectent des roches rigides et fracturées tels que les calcaires, les grès, ou les roches cristallines. iii. Les coulées boueuses constituent 8,24\%; elles se localisent au niveau des terrains argileux, ou argileux sableux. iiii. Les effondrements constituent $17,23 \%$ du nombre total des mouvements développés surtout au niveau des calcaires de la dorsale Les mouvements de terrain dans la région de Beni Idder, présent 10.10 $\%$ de la surface total de la région (Tab. 1).

Tableau 1. Pourcentages en nombre et en surface des quatre types de mouvements de terrain de la région d'étude

\begin{tabular}{|c|c|c|c|c|}
\hline Types & $\dot{\tilde{\Sigma}}$ & 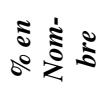 & 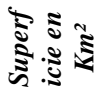 & 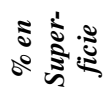 \\
\hline Glissements & 115 & 43,07 & 28,65 & 44,66 \\
\hline Eboulements & 84 & 31,46 & 10,92 & 17,01 \\
\hline $\begin{array}{c}\text { Coulées } \\
\text { Boueuses }\end{array}$ & 22 & 8,24 & 7,68 & 11.97 \\
\hline Effondrements & 46 & 17,23 & 16,91 & 26,36 \\
\hline
\end{tabular}

\section{Outils et méthode ;}

\subsection{La théorie de l'évidence}

La théorie de l'évidence (Weight of evidence) est l'approche que nous avons adoptée dans notre étude. C'est une version log-linéaire du théorème général de Bayes utilisant les principes de calcul de probabilité fondés sur les notions de probabilité a priori et de probabilité a posteriori [9]. Le principe de la méthode consiste à définir une relation mathématique entre les facteurs de prédisposition (variable prédictive, $V p$ ) et l'occurrence spatiale des glissements de terrain à travers ses données historiques (variable dépendante, $V d=V M$ ) [1]. Elle suppose trois hypothèses : $\mathbf{1}$. Les glissements de terrain potentiels se déclencheront selon les mêmes conditions que dans le passé, 2. Les facteurs de prédisposition $(V e=V P)$ sont connus apriori et introduits dans l'analyse, 3. L'ensemble des glissements de terrain est inventorié sur l'aire d'étude.

La méthode est fondée sur le calcul d'un poids positif $(1: W+)$ et d'un poids négatif $(2: W-)$, dont les valeurs dépendent de l'association entre $V p$ et $V d$. Ce ci pour calculer la probabilité a priori et la probabilité a posteriori. La probabilité a priori, qui est la probabilité qu'une unité de terrain (maille de calcul, 
un pixel) contienne une variable dépendante $(V d=V M)$, dans notre cas les glissements de terrain), est calculée suivant la densité sur la zone d'étude. La probabilité a posteriori est estimée après le calcul de la probabilité a priori selon la densité de $V d(V M)$ pour chaque variable considérée comme prédictive ( $V p$, dans notre cas chaque facteur de prédisposition) [1] [2] [3]. .

(1)

$$
W^{+}=\ln \frac{P\{B \mid E\}}{P\{B \mid \bar{E}\}}
$$$$
\text { (2) } W^{-}=\ln \frac{P\{\bar{B} \mid E\}}{P\{\bar{B} \mid \bar{E}\}}
$$

\section{B étant la classe de $\mathrm{Vp}$}

Le ratio représente la probabilité de présence sur la probabilité d'absence d'un phénomène. Les pondérations sont additionnées en utilisant le logarithme naturel des ratios appelé logit. Pour chaque VP, deux poids sont calculés, un positif $\left(\mathrm{W}^{+}\right)$et un autre négatif $\left(\mathrm{W}^{-}\right)$dont les valeurs dépendent de la relation spatiale entre les VP et la VM survenu dans le passé [9]. Lorsque plusieurs $V p$ sont combinées, les zones (mailles de calculs) qui ont un poids respectivement élevé ou faible correspondent respectivement à une forte ou faible probabilité de présence de $V M$.

\subsection{Outils de travail ;}

Les données nécessaires à cette étude ont été obtenues à partir des cartes topographiques existantes, des cartes géologiques et des images satellitaires en utilisant des techniques de traitement d'image. Toutes les fonctions de localisation, géologiques et géomorphologiques fournis par les différentes cartes thématiques ont été numérisées en utilisant le logiciel ArcGIS, puis transférées dans ArcView pour les analyses ultérieures. Ces données ont été regroupées en 5 groupes de variables : 1. un inventaire des mouvements de terrain, 2. Des paramètres topographiques (MNT, gradient de pente, expositions), 3. Des paramètres géologiques (lithologie, failles), 4. Le réseau hydrographique (densité du réseau de drainage), 5. L'occupation du sol.

\section{Facteurs prédictifs}

La base de données multi-sources élaborée (sous forme de cartes) des facteurs de prédisposition de l'occurrence spatiale des mouvements de terrain comporte deux variables géologiques: lithologie, fracturation; trois variables géométriques : cartes hypsométrique, des pentes et d'exposition un variable topographique: le réseau hydrographique et l'occupation du sol. Toutes les cartes élaborées ont été rastérisées avec la même taille du Pixel qui est de l'ordre de $25 \mathrm{~m}$.

\subsection{La lithologie}

La carte lithologique qu'on a réalisée présente cinq ensembles lithologiques ou cinq classes à savoir : les dolomies et calcaires, les marnes, les grès, les schistes et les alternances marno-calcaires. (Fig.4)

La fréquence des glissements au niveau des Grès, des marno-calcaires et Marnes montre une fréquence relativement élevée, et au niveau des marno-calcaires elle est jugée très élevée.

\subsection{Occupation des sols}

L'occupation du sol est obtenue par analyse et interprétation d'images satellitaires et de photographies aériennes. Nous avons définit cinq classes : 1. La forêt qui présente $33,2 \%$ de la surface totale, 2. La végétation naturelle avec une surface de $26 \%, 3$. terres arables de $22,9 \%, 4$. terrains agricoles avec $17,3 \%$ de la surface, 5. terrains nus de $0,6 \%$ (Fig.5).

La corrélation entre l'occupation du sol et la fréquence des glissements de terrain montre que la fréquence des glissements de terrain est relativement faible sur les terrains nus et plus élevé sur des terres arables et forêts.

\subsection{Le gradient de pente}

On a réalisé la carte des pentes à partir du MNT de la Région de Beni Idder avec cinq classes bien déterminer : classe $1\left(0^{\circ}-5^{\circ}\right)$, classe $2\left(5^{\circ}-15^{\circ}\right)$, classe $3\left(15^{\circ}-25^{\circ}\right)$, classe $4\left(25^{\circ}-45^{\circ}\right)$ et classe 5 $\left(45^{\circ}-89^{\circ}\right)$ (Fig.6).

L'examen de la répartition des glissements de terrain avec les classes de gradient de pente, montre une augmentation de la fréquence des glissements de terrain dans trois classes de gradient de pente où la fréquence maximale est atteinte dans la classe $5^{\circ}$ $15^{\circ}$.

\subsection{Hypsométrie}

On a réalisé la carte d'hypsométrie avec les classes suivantes : $33 \mathrm{~m}-400 \mathrm{~m}, 400 \mathrm{~m}-650 \mathrm{~m}, 650 \mathrm{~m}-$ 900m, 900m- $1250 \mathrm{~m}$ et $1250 \mathrm{~m}-1913 \mathrm{~m}$ (Fig 7). Au nveau des altitudes de $400 \mathrm{~m}$ à $900 \mathrm{~m}$ la fréquence des mouvements de terrains est très élevée, on note aussi que la fréquence des mouvements de terrain est assez importante aux altitudes inférieures à $400 \mathrm{~m}$.

\subsection{Exposition des versants}

La carte d'exposition a été reclassifiée en cinq classes importantes (Fig.8). La répartition des glissements de terrain cartographiées par rapport aux classes des expositions, montre que les glissements sont relativement plus fréquents au niveau des orientés Nord et Ouest. 


\subsection{La fracturation}

L'activité tectonique récente et héritée peut intervenir, en conditionnant les mouvements de terrain dans les zones où les terrains sont fragilisés [10] [11]. Ainsi la cartographie des mouvements de terrain dans la région d'étude, montre qu'un nombre important de mouvements s'est développé le long des principaux accidentsde direction NS et les fronts de chevauchements de direction, , etc). Ces diverses failles guident un certain nombre d'écoulements de surface. Elles montrent aussi une relation avec la répartition des sources d'eau [10] [11] [12]. (Fig.9)
La relation entre la fréquence des glissements de terrain et la distance aux failles, montre que la fréquence des glissements de terrain diminue lorsque la distance aux failles augmente. Avec le maximum des mouvements de terrain se trouve à $100 \mathrm{~m}$ des failles.

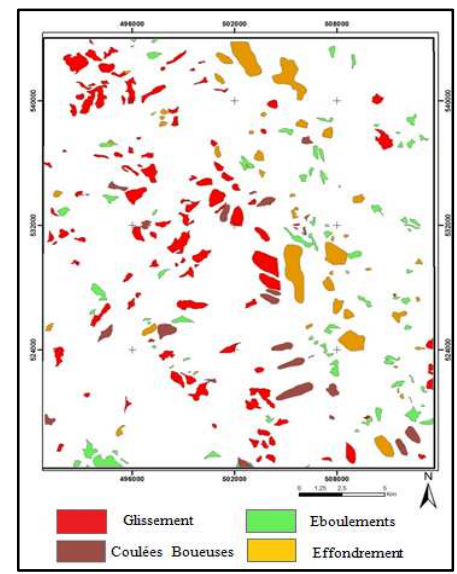

Fig.3 carte d'inventaire des mouvements de terrain.

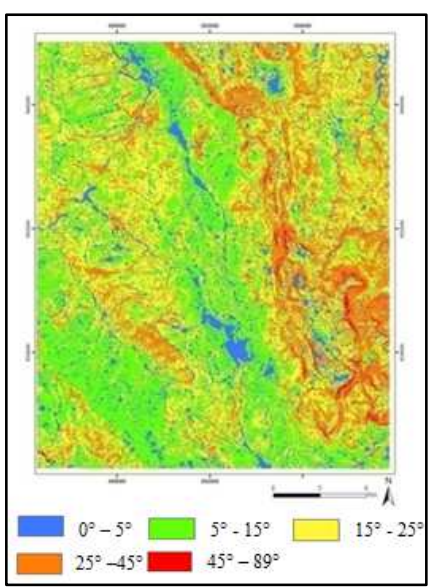

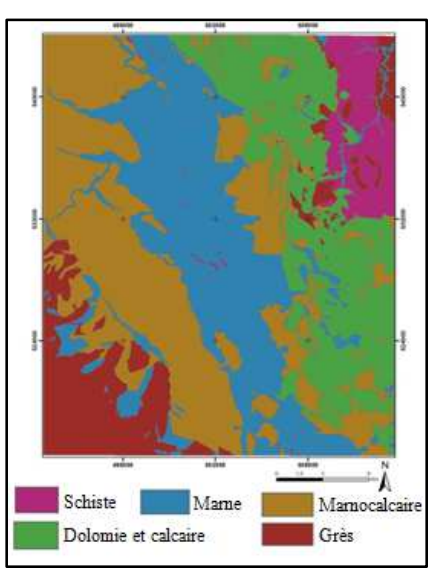

Fig.4 Carte des différentes formations lithologiques.

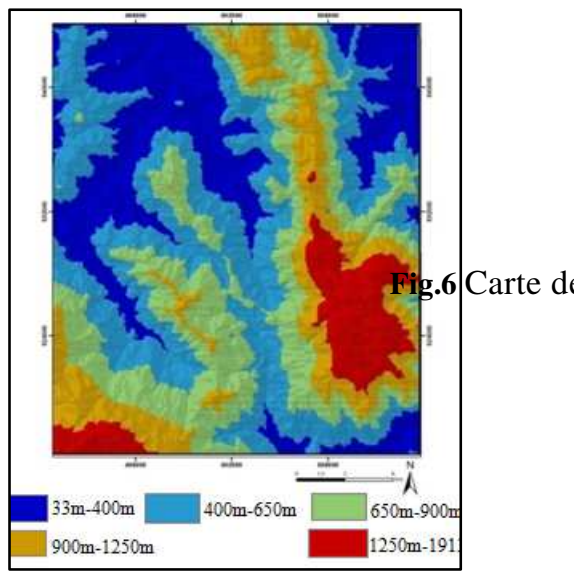

Fig.7 Carte d'hypsométrie (en mètre).

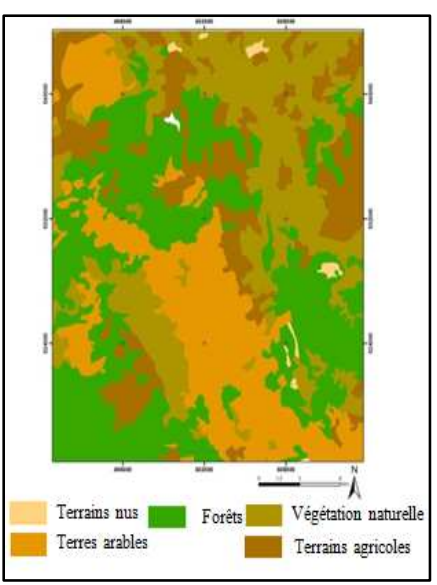

Fig.5 Carte d'occupation des sols.

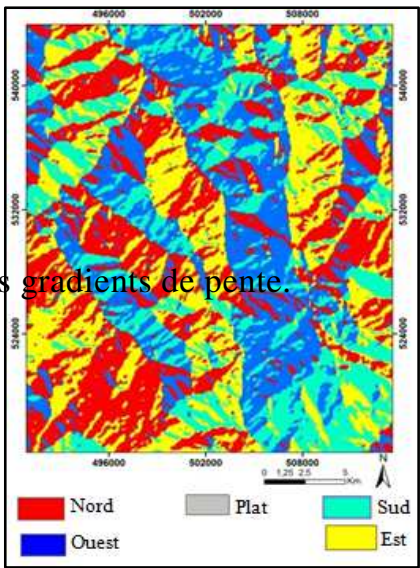

Fig.8 Carte d'expositions.

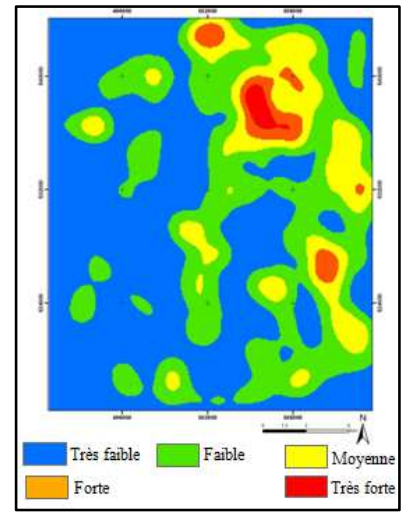

Fig.10 Carte de la densité du réseau hydrographique.

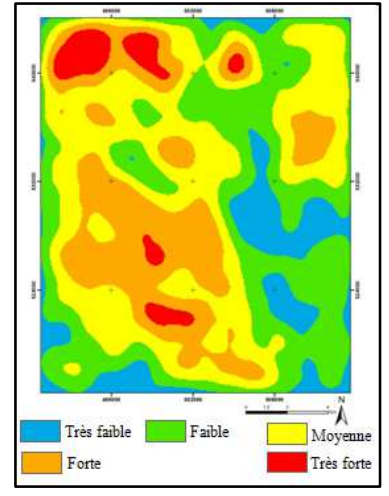

Fig.11 Carte de densité du réseau hydrographique 


\section{Réseau hydrographique}

Le réseau hydrographique est pris en considération pour deux raisons: i. La nature imperméable de la majorité des terrains dans le Rif (à l'exception de la dorsale calcaire), se traduit par un écoulement de surface très important lors de la période pluviale et à chaque orage ce qui conduit soit à une érosion soit à une infiltration à travers des zones fragiles (stratification inclinée, schistosité, failles etc...) contribuant à une diminution de la résistance au cisaillement des matériaux; ii. Son analyse en terme d'anomalies et de perturbation peut nous renseigner dans les zones dépourvues de terrain plio-quaternaire de notre secteur d'étude de la réactivation récente des failles [10] [12]. (Fig.10) La relation entre la fréquence des glissements de terrain et la densité du réseau hydrographique, montre que la fréquence des glissements de terrain augmente dans la classe ou la distance entre 200 et $400 \mathrm{~m}$.

\section{Résultats et analyses}

\subsection{Calcul du poids ;}

Les cartes des paramètres de prédisposition combinées avec la carte de glissement de terrain permettent de calculer les poids positifs et négatifs et les contrastes, comme indiqué dans le tableau.2.

Les valeurs positives du contraste (C) enregistrés pour les paramètres lithologiques (Marno-calcaire, Marne et dolomie-calcaire), l'occupation du sol (terres arables et terrains nus), distance aux failles (inférieure à $300 \mathrm{~m}$ ), attestent de leur rôle significatif dans l'apparition des mouvements de terrain.

Tableau 2: Corrélation spatiale entre les variables prédictives et les valeurs de pondération calculées par la théorie de l'évidence, $(\mathrm{W}+)$ : la valeur la probabilité a priori, (W-) : la valeur de la probabilité à posteriori, (C) : le contraste qui est la différence entre $\mathrm{W}+$ et $\mathrm{W}-, \mathrm{S}(\mathrm{C})$ : la variance du contraste

\begin{tabular}{|c|c|c|c|c|c|}
\hline $\begin{array}{c}\text { Formations } \\
\text { géologiques }\end{array}$ & $\begin{array}{c}\text { Surface } \\
\text { en } \mathbf{K m}^{2}\end{array}$ & $\boldsymbol{W +}$ & $\boldsymbol{W}$ - & $\boldsymbol{C}$ (Contraste) & $\boldsymbol{S ( C )}$ \\
\hline Grès & 73,443 & $-0,4067$ & 0,0436 & $-0,4503$ & 0,5246 \\
\hline $\begin{array}{c}\text { Dolomie et } \\
\text { calcaires }\end{array}$ & 130,64 & 0,0368 & $-0,0099$ & 0,0466 & 0,3441 \\
\hline $\begin{array}{c}\text { Marno- } \\
\text { calcaires }\end{array}$ & 212,39 & 0,0430 & $-0,0227$ & 0,0657 & 0,2961 \\
\hline Schistes & 37,478 & $-1,1347$ & 0,0431 & $-1,1778$ & 1,0135 \\
\hline Marnes & 173,90 & 0,1915 & $-0,0836$ & 0,2751 & 0,3005 \\
\hline $\begin{array}{c}\text { Distance par } \\
\text { rapport aux } \\
\text { Failles }\end{array}$ & $\begin{array}{c}\text { Surface } \\
\text { en } \mathbf{K m}^{2}\end{array}$ & $\boldsymbol{W +}$ & $\boldsymbol{W}$ - & $\boldsymbol{C}($ Contraste $)$ & $\boldsymbol{S}(\boldsymbol{C})$ \\
\hline 100 & 332,40 & 0,0498 & $-0,0621$ & 0,1120 & 0,2856 \\
\hline 200 & 196,47 & 0,0650 & $-0,0310$ & 0,0960 & 0,3002 \\
\hline 300 & 70,891 & $-1,0694$ & 0,0813 & $-1,1508$ & 0,7242 \\
\hline 400 & 16,292 & 0,4266 & $-0,0141$ & 0,4407 & 0,7326 \\
\hline$>500$ & 4,4508 & 1,0577 & $-0,0130$ & 1,0707 & 1,0392 \\
\hline
\end{tabular}

\begin{tabular}{|c|c|c|c|c|c|}
\hline $\begin{array}{c}\text { Gradient de } \\
\text { pente }\end{array}$ & $\begin{array}{l}\text { Surface } \\
{\text { en } \mathbf{K m}^{2}}^{2}\end{array}$ & W+ & $W-$ & $C($ Contraste $)$ & $S(C)$ \\
\hline $0-5^{\circ}$ & 67,686 & 0,6162 & $-0,1063$ & 0,7225 & 0,3587 \\
\hline $5-15^{\circ}$ & 219,90 & 0,0598 & $-0,0339$ & 0,0938 & 0,2927 \\
\hline $15-25^{\circ}$ & 213,09 & $-0,5493$ & 0,1967 & $-0,7459$ & 0,3553 \\
\hline $25-45^{\circ}$ & 118,28 & 0,1250 & $-0,0318$ & 0,1568 & 0,3443 \\
\hline $45-89^{\circ}$ & 8,0862 & 0,3906 & $-0,0064$ & 0,3971 & 1,0252 \\
\hline $\begin{array}{c}\text { Exposition } \\
\text { des versants }\end{array}$ & $\begin{array}{l}\text { Surface } \\
\text { en } \mathrm{Km}^{2}\end{array}$ & $W+$ & $W-$ & $C($ Contraste $)$ & $S(C)$ \\
\hline Plat & 37,194 & 0,9925 & $-0,1107$ & 1,1033 & 0,3946 \\
\hline Nord & 241,45 & $-0,3411$ & 0,1670 & $-0,5082$ & 0,3165 \\
\hline Est & 83,763 & $-0,3121$ & 0,0406 & $-0,3527$ & 0,4746 \\
\hline Sud & 77,692 & 0,1046 & $-0,0157$ & 0,1202 & 0,4115 \\
\hline Ouest & 187,01 & 0,1115 & $-0,0514$ & 0,1628 & 0,3003 \\
\hline $\begin{array}{l}\text { Distance au } \\
\text { réseau } \\
\text { hydro- } \\
\text { graphique }\end{array}$ & $\begin{array}{l}\text { Surface } \\
\text { en } \mathbf{K m}^{2}\end{array}$ & $W+$ & $W$ - & $C($ Contraste $)$ & $S(C)$ \\
\hline 100 & 69,838 & 0,2128 & $-0,0300$ & 0,2429 & 0,4119 \\
\hline 200 & 190,34 & $-0,2555$ & 0,0939 & $-0,3494$ & 0,3330 \\
\hline 300 & 205,51 & $-0,1038$ & 0,0467 & $-0,1505$ & 0,3103 \\
\hline 400 & 137,97 & 0,0737 & $-0,0216$ & 0,0953 & 0,3337 \\
\hline$>500$ & 26,157 & 0,8868 & $-0,0620$ & 0,9487 & 0,4819 \\
\hline $\begin{array}{c}\text { Occupation } \\
\text { des sols }\end{array}$ & $\begin{array}{l}\text { Surface } \\
\text { en } \mathbf{K m}^{2}\end{array}$ & $W_{+}$ & $W$ - & $C($ Contraste $)$ & $S(C)$ \\
\hline Forêts & 218,00 & $-0,3595$ & 0,1475 & $-0,5070$ & 0,3211 \\
\hline $\begin{array}{l}\text { Végétation } \\
\text { naturelle }\end{array}$ & 161,96 & 0,0152 & $-0,0053$ & 0,0205 & 0,3139 \\
\hline $\begin{array}{c}\text { Terres } \\
\text { arables }\end{array}$ & 143,20 & 0,2774 & $-0,0971$ & 0,3745 & 0,3019 \\
\hline $\begin{array}{c}\text { Terrains } \\
\text { agricoles }\end{array}$ & 107,76 & $-0,0204$ & 0,0041 & $-0,0245$ & 0,3690 \\
\hline $\begin{array}{c}\text { Terrains } \\
\text { nus }\end{array}$ & 3,6054 & 1,9718 & $-0,0326$ & 2,0044 & 0,7732 \\
\hline $\begin{array}{l}\text { L'hypso- } \\
\text { métrie }\end{array}$ & $\begin{array}{c}\text { Surfac } \\
\text { e en } \\
\mathrm{Km}^{2} \\
\end{array}$ & $W+$ & $W$ - & C(Contraste $)$ & $S(C)$ \\
\hline $33-400 \mathrm{~m}$ & 148,20 & 0,0073 & $-0,0022$ & 0,0095 & 0,3335 \\
\hline $\begin{array}{c}400-650 \\
\mathrm{~m}\end{array}$ & 190,28 & 0,2217 & $-0,1116$ & 0,3334 & 0,2929 \\
\hline $\begin{array}{c}650-900 \\
m\end{array}$ & 191,37 & $-0,0259$ & 0,0110 & $-0,0369$ & 0,3104 \\
\hline $\begin{array}{c}900-1250 \\
\mathrm{~m}\end{array}$ & 70,632 & $-1,0567$ & 0,0797 & $-1,1364$ & 0,7242 \\
\hline $\begin{array}{l}1250- \\
1913 \mathrm{~m}\end{array}$ & 33,887 & 0,0985 & 0,0124 & $-0,2608$ & 0,3255 \\
\hline
\end{tabular}

\subsection{Calcul de l'indépendance conditionnel (IC)}

\subsubsection{Degré de liberté entre chaque paire de variables de predisposition}

Parmi les sept variables de predisposition traités, six ont été retenues (occupation des sols, la pente, le facies, hypsométrie, distance aux failles et le réseau hydrographique) étant donné que les valeurs du degré de liberté $(\boldsymbol{\alpha})$ entre chaque paire de variables obtenue par la méthode de WofE (tableau.4) sont supérieurs à $\mathbf{0 , 0 5}$ $(\boldsymbol{\alpha}>\mathbf{0 , 0 5})$ [1] [2] [3]. La variable exposition a été retirée $(\alpha<0,05)$ étant donné sa dépendance avec la variable pente. Ce résultat a été confirmé par le test de chi-carrée $\left(\mathrm{X}^{2}\right)$ etant donné que l'ecart n'est pas important entre 
l'écart de $\mathrm{X}^{2}$ observé et $\mathrm{X}^{2}$ théorique pour chaque couple de facteurs et pour l'ensemble des variables à modéliser.

Tableau 3 : Valeurs du degré de liberté $\alpha$ entre chaque paire de variables

\begin{tabular}{|c|c|c|c|c|c|}
\cline { 2 - 6 } \multicolumn{1}{c|}{} & $\begin{array}{c}\text { Occupa } \\
\text {-tion } \\
\text { des } \\
\text { sols }\end{array}$ & Pente & Facies & $\begin{array}{c}\text { Hypso- } \\
\text { métrie }\end{array}$ & $\begin{array}{c}\text { Réseau } \\
\text { hydro- } \\
\text { graphique }\end{array}$ \\
\hline $\begin{array}{c}\text { Distance } \\
\text { au faille }\end{array}$ & $\begin{array}{c}0,991 \\
9\end{array}$ & 0,8358 & 0,3607 & 0,9996 & 0,9330 \\
\hline $\begin{array}{c}\text { Occupa- } \\
\text { tion des } \\
\text { sols }\end{array}$ & & 0,9841 & 0,8133 & 0,3840 & 0,9611 \\
\hline Pente & & & 0,8392 & 0,9736 & 0,9952 \\
\hline Facies & & & & 0,8091 & 0,6982 \\
\hline $\begin{array}{c}\text { Hypso- } \\
\text { métrie }\end{array}$ & & & & & 0,7724 \\
\hline
\end{tabular}

\subsubsection{La carte de susceptibilité aux mouvements de terrain de Beni Idder (WofE)}

La carte obtenue permet de distinguer 5 classes de susceptibilité : très forte, forte, moyenne, faible, et très faible (Fig.11).

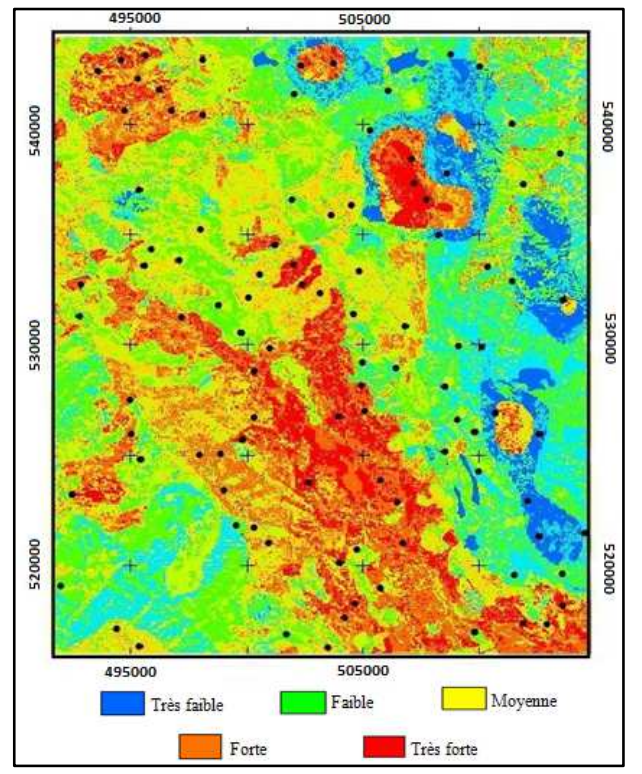

Fig.11 La carte de susceptibilité aux mouvements de terrain par la théorie de l'évidence de la région de Tleta de Beni Ider

Pour la validation de la carte de susceptibilité aux mouvements de terrain de la région de Tleta de Beni Ider, on a superposé les mouvements de terrain cartographiés sur la carte de susceptibilité obtenue afin de définir la proportion des mouvements de terrain par classes de susceptibilité. Le résultat statistique en proportion des mouvements de terrain par classes de susceptibilité a montré que $53 \%$ des mouvements de terrain cartographiés se situent dans la zone à très forte à forte susceptibilité, $24 \%$ des mouvements de terrain dans la zone moyenne.
Tableau. 4 : Les pourcentages des mouvements de terrain par rapport les classes de susceptibilité

\begin{tabular}{|l|c|}
\hline \multicolumn{1}{|c|}{ Susceptibilité } & Pourcentage des MT (\%) \\
\hline Très forte & 29 \\
\hline Forte & 24 \\
\hline Moyenne & 20 \\
\hline faible & 15 \\
\hline très faible & 12 \\
\hline
\end{tabular}

\section{Conclusion}

L'analyse de la susceptibilité aux mouvements de terrain dans la région montagneuse de Beni Idder a été facilitée grâce à l'utilisation d'outils de la géomatique (Télédétection, SIG, GPS, etc.).

La théorie de l'évidence a été testée dans la région de Tétouan-Chefchaouen (Rif septentrional) pour évaluer la susceptibilité liée aux quatre types de mouvements de terrain. Les facteurs conditionnellement indépendants ont été retenus et intégrés dans le processus de modélisation afin de produire les cartes de probabilité d'occurrence de mouvements de terrain.

Les facteurs de prédisposition de l'occurrence spatiale des mouvements de terrain, ont été rastérisés avec la même taille du Pixel qui est de l'ordre de $25 \mathrm{~m}$, puis classés en 5 classes. Les mouvements de terrain significatifs et représentatifs ont été identifiés et cartographiés.

Le test chi-carré $\mathrm{X}^{2}$ a permis de définir les facteurs prédictifs conditionnellement indépendants vis à vis aux mouvements de terrain VM. Les poids positifs et négatifs ainsi que leurs contrastes ont été calculés pour chaque classe de variables. Ce calcul a permis d'identifier les variables qui sont positivement corrélées $\left(W^{+}>0\right.$ et $\left.W^{-}<0\right)$ ou négativement corrélées $\left(W^{+}<0\right.$ et $\left.W^{-}>0\right)$.

La carte de susceptibilité élaborée fait ressortir que : i. Les glissements de terrain sont les plus fréquents et se localisent sur des terrains marneux à marno-calcaires ; ii. Les éboulements viennent par la suite en terme de fréquence et se localisent au niveau de la dorsale calcaire (dolomie calcaire, marno-calcaire) ; iii. Les coulées boueuses se localisent dans les zones où dominent les marnes; iii. Les effondrements sont situés essentiellement au centre de la dorsale calcaire ce qui est justifié par sa nature lithologique. Ainsi les zones les plus exposées aux instabilités de terrains se localisent au niveau de la dorsale calcaire et des nappes des flyschs.

Les résultats statistiques montrent que $53 \%$ des mouvements de terrain cartographiés se situent dans la zone à très forte à forte susceptibilité, $24 \%$ des mouvements de terrain dans la zone moyenne.

La carte de susceptibilité aux mouvements de terrain élaborée, servira dans l'aménagement et le 
développement durable de la région, ceci pour une éventuelle extension urbaine, ou lors des planifications de nouveaux tracés de routes et autoroutes. Elle constitue un document utile pour la prévention et l'information des populations aux risques de mouvements de terrain dans la région de Tleta de Beni Ider.

\section{References}

1. A. Carrara , M. Cardinali, F. Guzzetti, P. Reichenbach, GIS-based techniques for mapping landslide hazard. Geographical Information System in assessing natural hazard(1995)

2. C.J. Van Westen, E.A. Castellanos Abella, L.K. Sekhar, Spatial data for landslide susceptibility, hazards and vulnerability assessment: an overview. In: Engineering geology. ( 2008)

3. Y. Thiery, J-P. Malet, S. Sterlacchini, A. Puissant, O. Maquaire, Landslide susceptibility assessment by bivariate methods at large scales: Application to a complex mountainous environment. Geomorphology. (2007)

4. M. El Moulat, L. Ait Brahim, M. Mastere and A. I. Jemmah, "Mapping of Mass Movements Susceptibility in the Zoumi Region Using Satellite Image and GIS Technology (Moroccan Rif)," International Journal of Scientific \& Engineering Research, vol. 6(2), pp. 210-217, (2015)

5. M. Mansour, L. Ait Brahim, P. Potherat, Z. Sekkat. "Etude du glissement de Bouhalla par une approche multisource (commune de Chaouene, Rif, Maroc)." Revue de Génie civil, 81, Mai 1999, pp.34-41, (1999).

6. A.El Gharbaoui, La terre et l'Homme dans la péninsule tingitane. Etude sur l'Homme et le milieu dans le Rif occidental. Travaux de l'Institut Scientifique, série Géologie et Géographie Physique, Rabat, 15, 439 p. (1981)

7. M. Moukhchane, S. Bouhlassa, A. Chalouan ; Approche cartographique et magnétique pour l'identification des sources de sédiments : cas du bassin versant Nakhla (Maroc). Sécheresse, 9 :227-32. (1998)

8. D.J. Varnes, Landslide Hazard Zonation, a review of principles and practice. IAEG Commission on Landslides. UNESCO, Paris. (1984)
9. G.F. Bonham-Carter, F.P. Agterberg, Weights of evidence modeling: a new approach to mapping mineral potential. Geological Survey of Canada Paper, 89-9. (1989)

10. L. Aït Brahim - Tectonique cassante et état de contaraintes récentes $d u$ Maroc Nord, contibution à l'étude du risque sismo-tectonique au Maroc. Thèse de doctorat d'état, Université Mohamed V, Faculté des Sciences, Rabat. (1991)

11. L. Aït Brahim, P. Chotin , S. Hinaj, A. Abdelouafi, A. El Adraoui , C. Nakcha, D. Dhont, M. Charroud, F. Sossey Alaoui, M. Amrhar, A. Bouaza, H. Tabyaoui, A. Chaouni. Paleostress evolution in the Moroccan African margin from Triassic to Present. Tectonophysics. (2002)

12. Deffontaines et al., Investigation of active faults in Moroco using morphometric methods and pattern analyses sonderdruck an Geologische Rundschau, 8, Helt 1, Stuttgart, Deutschland, P. 199-210, (1992). 